\title{
Presentation of Severe Rheumatic Mitral Stenosis at the Peak of the COVID-19 Pandemic and the Presumptive Treatment as Severe Coronavirus Illness
}

\author{
Christopher Balfe $^{1}$, Cormac O'Connor ${ }^{1}$, Gerard Giblin², Kevin Walsh², Ivan Casserly², David Moore ${ }^{1}$, Vincent Maher ${ }^{1}$ \\ ${ }^{1}$ Department of Cardiology, Tallaght University Hospital, Tallaght, Dublin, Ireland \\ ${ }^{2}$ Department of Cardiology, Mater Misericordiae University Hospital, Dublin, Ireland
}

Received: $23 / 08 / 2020$

Accepted: $23 / 09 / 2020$

Published: $04 / 11 / 2020$

How to cite this article: Balfe C, O'Connor C, Giblin G, Walsh K, Casserly I, Moore D, Maher V. Presentation of severe rheumatic mitral stenosis at the peak of the COVID-19 pandemic and the presumptive treatment as severe coronavirus illness. EJCRIM 2020;7: doi:10.12890/2020_001957.

Conflicts of Interests: The Authors declare that there are no competing interests.

This article is licensed under a Commons Attribution Non-Commercial 4.0 License

\section{ABSTRACT}

This case report describes a young female Caucasian patient with newly presenting severe mitral stenosis at the peak of the coronavirus pandemic in the Republic of Ireland.

The initial presumptive diagnosis was of severe coronavirus illness. This case report highlights the importance of keeping an open mind to alternative diagnoses and examines some of the challenges in the diagnosis and management of a rare condition in the pandemic environment. This patient gained $10 \mathrm{~kg}$ of weight within 6 weeks of percutaneous balloon mitral valvuloplasty, highlighting the contribution of cardiac cachexia to her low body weight and demonstrating the exceptional benefit that this treatment can offer to patients.

\section{LEARNING POINTS}

- Tohighlight mitral stenosis as a cause of cardiaccachexia and to examine the benefits gained from percutaneous balloon commissurotomy.

- To highlight the potential for other serious conditions to masquerade as COVID-19 and the importance of keeping an open mind to diagnoses.

- To examine the use of bedside echocardiography in the patient presenting with presumed coronavirus illness.

\section{KEYWORDS}

Mitral stenosis, rheumatic heart disease, valvular heart disease

\section{CASE DESCRIPTION}

We present the case of an 18-year-old Caucasian female admitted on 8 April 2020 with a 10-day history of progressive dyspnoea, dry cough and pleuritic chest pain. The patient had a background history notable only for anorexia nervosa, with a body weight of $40 \mathrm{~kg}$.

On arrival to the emergency department she was pyrexial and tachycardic, but normotensive. General inspection was significant for the presence of low body mass index and tachypnoea. Vital signs on admission were as follows: heart rate 145 bpm, blood pressure $114 / 79$ $\mathrm{mmHg}$, temperature $37.8^{\circ} \mathrm{C}$, respiratory rate 22 and saturation $96 \%$ on room air.

Chest auscultation revealed widespread bilateral inspiratory and expiratory crepitations. No murmur was identified and there was no ankle oedema. 
Blood results included an elevated white cell count of $15.6 \times 10^{9} / \mathrm{l}$. C-reactive protein levels were elevated at $68 \mathrm{mg} / \mathrm{l}$. Renal function was normal with creatinine levels of $66 \mu \mathrm{mol} / \mathrm{l}$ and urea levels of $4.2 \mathrm{mmol} / \mathrm{l}$. Liver function, corrected calcium and magnesium were all within the normal range. High-sensitivity troponin testing and CK levels were normal but NT-proBNP was elevated at 1,916 pg/ml.

12-lead ECG revealed sinus tachycardia with right axis deviation and a heart rate of $150 \mathrm{bpm}$ and tall peaked P waves, especially notable in lead II.

The initial portable erect chest x-ray demonstrated severe bilateral perihilar consolidation, particularly in the upper and mid zones. There was no pleural effusion.

The initial presumptive diagnosis was COVID-19 pneumonia with accompanying systemic inflammatory response syndrome.

Over the first 12 hours of the patient's admission her sinus tachycardia persisted at 140-150 bpm. She had a further 3 pyrexial episodes above $37.5^{\circ} \mathrm{C}$. Several fluid boluses were administered, amounting in total over these 12 hours to $1,500 \mathrm{ml}(37.5 \mathrm{ml} / \mathrm{kg})$.

While the patient initially presented with adequate saturation on room air, her oxygen requirement increased, eventually to the point of requiring $60 \% \mathrm{FiO} 2$ via a non-rebreather mask. In parallel to this, the patient became increasingly tachypnoeic and distressed, in particular when lying flat.

A repeat chest $x$-ray at this point revealed worsening extensive perihilar consolidation bilaterally (Fig. 1).

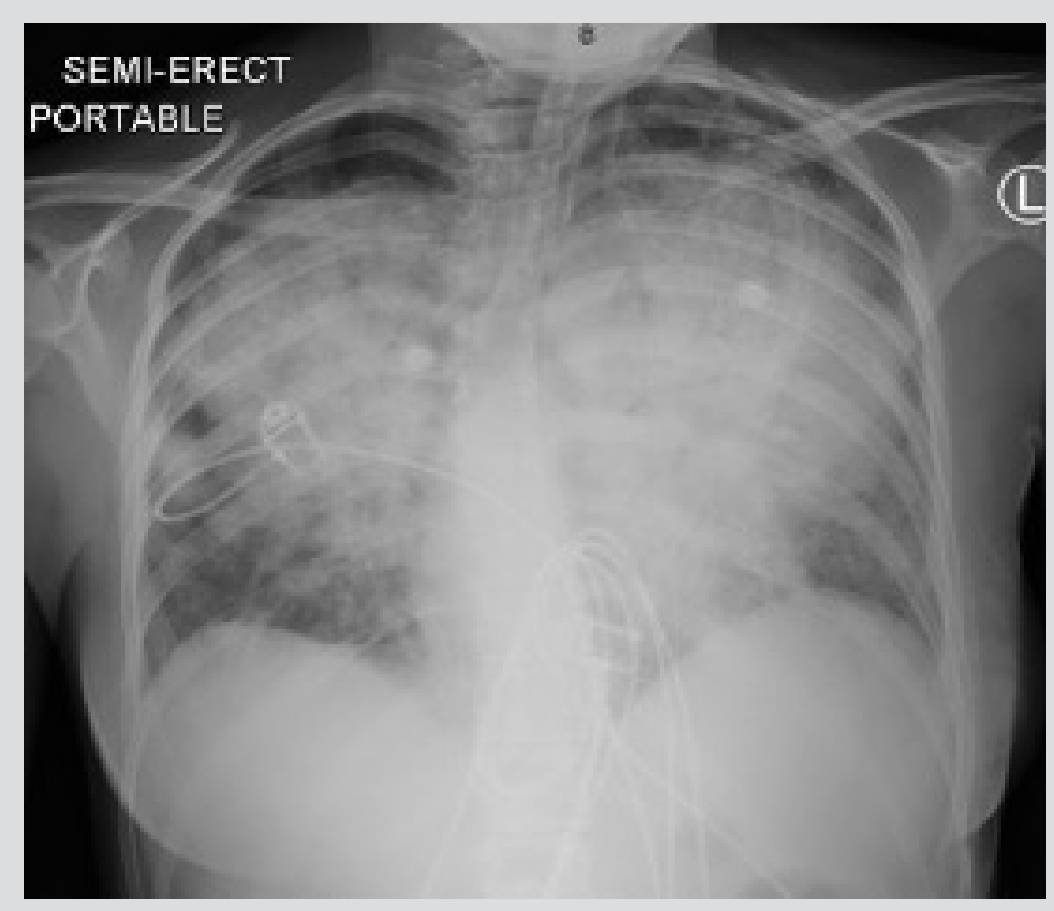

Figure 1. A repeat chest $x$-ray 12 hours after arrival

At this point a request was made for a bedside echocardiogram. This study was limited by tachycardia and severe orthopnoea, but demonstrated classical mitral stenosis (Figs. 2 and 3).

There was thickening of the mitral valve leaflets, most marked at the tips, with doming of the anterior mitral valve leaflet during diastole (Fig. 2). The left atrium was severely dilated, measuring $5.62 \mathrm{~cm}$ with a volume of $116.6 \mathrm{ml}$. No mitral regurgitation was noted. The left ventricle was grossly normal in size with a normal ejection fraction. The right ventricle was mildly dilated with normal to supernormal function. Right atrial size was normal.

The following data was obtained, but was interpreted with caution in view of the profound effect of the elevated heart rate on mitral valve parameters: the mean mitral valve gradient was estimated at $21 \mathrm{mmHg}$ with a peak gradient up to $34 \mathrm{mmHg}$. Using Doppler pressure halftime data, the mitral valve area was estimated at $0.9 \mathrm{~cm}^{2}$. The maximum tricuspid regurgitant pressure gradient was found to measure up to $85 \mathrm{mmHg}$.

The patient had never undergone echocardiography in the past and this study was therefore newly diagnostic of significant mitral stenosis with accompanying pulmonary hypertension. 

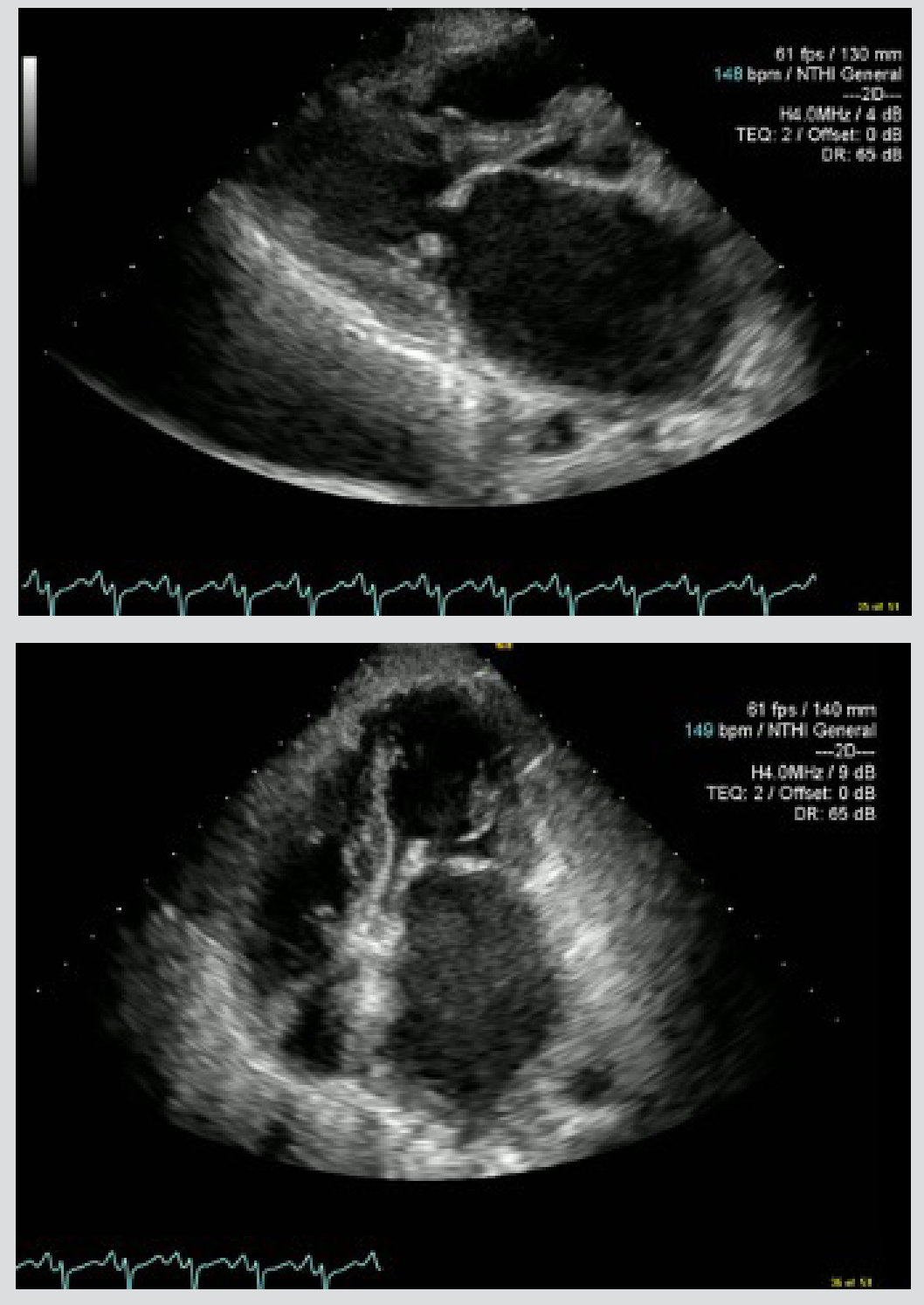

Figure 2. Parasternal long axis view with a markedly dilated left atrium and doming of the anterior mitral valve leaflet during diastole

Figure 3. Apical 4 chamber view taken during atrial systole demonstrating a severely dilated left atrium and a markedly abnormal mitral valve

Subsequent collaborative management between the Cardiology and Critical Care team took place in the Intensive Therapy Unit with full coronavirus precautions. The patient was placed on a continuous positive airway pressure mask with a PEEP of $10 \mathrm{~cm} \mathrm{H}_{2} \mathrm{O}$ and an initial $\mathrm{FiO} 2$ of $60 \%$. An arterial catheter and internal jugular central venous line were inserted.

Heart rate control was prioritized to prolong diastole and improve left ventricular filling. As such, an esmolol infusion was initiated. Weightbased diuresis with intravenous furosemide was commenced.

Of note, when diuresis was commenced, the central venous pressure was found to be between 4 and $6 \mathrm{mmHg}$. This was attributed to the presence of supernormal right ventricular function and tachycardia with shunting of extra volume into the lungs, bringing about a disconnect between the right and left ventricular filling pressures.

Progress over the subsequent 4 days was as follows: The patient was transferred to an Adult Congenital Heart Disease specialist centre. She was ultimately intubated and ventilated and required vasopressor support due to refractory pulmonary oedema. On 13 April 2020 the patient underwent percutaneous mitral balloon commissurotomy (PMBC) (Fig. 4).

Three coronavirus swabs were sent - all were negative.

Detailed history from the patient's mother propounded an episode of possible Sydenham's chorea at the age of 8 years.

The post-procedural transthoracic echo showed a mean mitral valve gradient of $7.78 \mathrm{mmHg}$ and improvement in the maximum tricuspid regurgitant gradient to approximately $32 \mathrm{mmHg}$ (Fig. 5) 


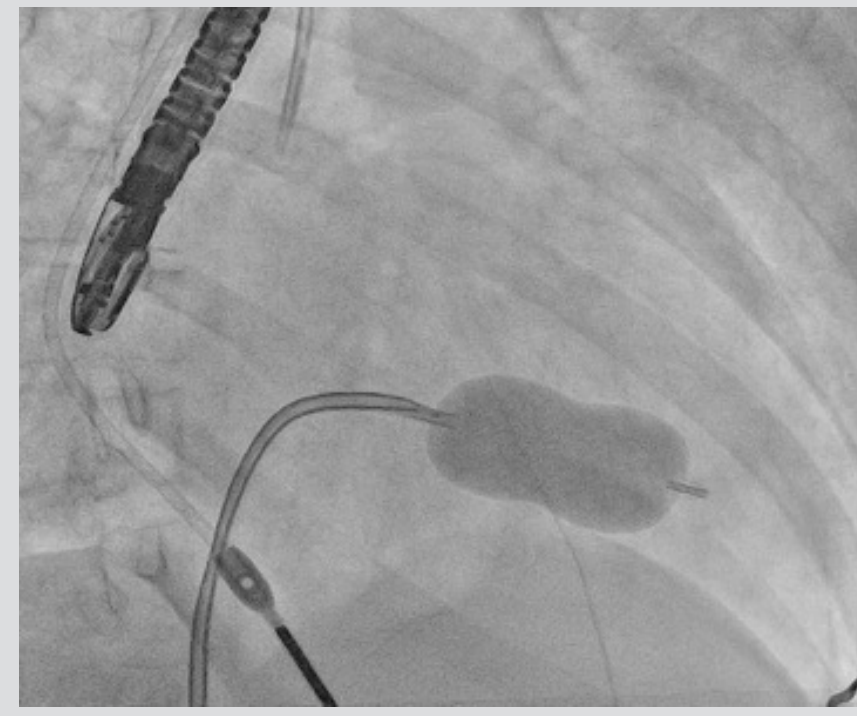

Figure 4. Percutaneous mitral commissurotomy using the Inoue balloon technique

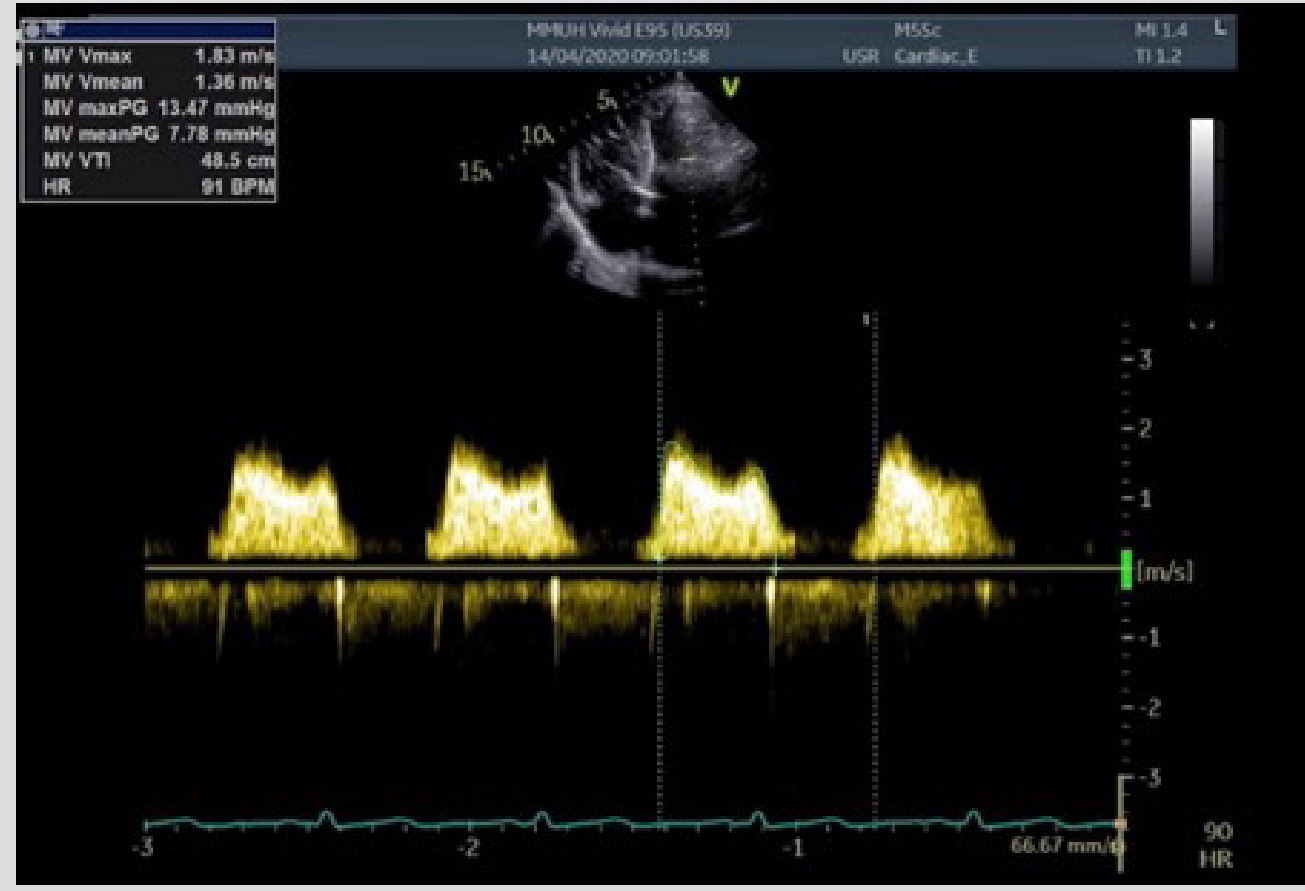

Figure 5. Apical 4 chamber view with CW Doppler demonstrating improvement in the MV mean gradient, carried out on Day 1 post-PMBC

Following PMBC, there was rapid clearing of the chest $x$-ray and improvement in clinical status, with extubation carried out the day after the procedure. The patient was discharged home with follow-up echocardiography and clinical review planned within 3 months.

\section{DISCUSSION}

Since the mid-20th century there has been virtual disappearance of acute rheumatic fever in developed countries, yet rheumatic heart disease remains endemic in Africa, Asia and South America. The estimated age-standardized prevalence of rheumatic heart disease in 2015 was 444 cases per 100,000 population for countries with an endemic pattern, and 3.4 cases per 100,000 population for industrialized countries $^{[1]}$.

Accordingly, the presence of severe rheumatic mitral stenosis in this patient was a surprising finding. Cardiac cachexia and a history of Sydenham's chorea can be features of the condition and were present in this patient. 
Symptoms may go unrecognized by patients with rheumatic mitral stenosis when slow progression of the condition causes insidious deconditioning and a gradual decline in exercise tolerance. Symptoms may be precipitated by increases in cardiac output, which raises transmitral flow, or tachycardia, which decreases diastolic filling time. Common precipitants of symptoms include pulmonary infection, atrial fibrillation and pregnancy.

Indeed, prior to this presentation, our patient had complained of no cardiorespiratory symptoms. Nevertheless, 6 weeks after undergoing PMBC, the patient had gained $10 \mathrm{~kg}$ in body weight and noticed a profound increase in energy.

The physical findings of severe mitral stenosis are subtle, and the murmur may be difficult to appreciate. Echocardiography is the cornerstone of diagnosis and the finding of a valve area of $<1.0 \mathrm{~cm}^{2}$ and a mean gradient $>10 \mathrm{mmHg}$ (at heart rates between $60-80 \mathrm{bpm}$ in sinus rhythm) is indicative of severe mitral stenosis.

Bedside echocardiography was invaluable to the care of the patient, who until this point was being empirically treated for coronavirus illness and in whom administration of IV fluids had contributed to a deteriorating respiratory status. The presence of ECG abnormalities, the persistence of a profound sinus tachycardia and an increasing oxygen requirement led to the request for a bedside echocardiogram. This case supports the judicious use of bedside echocardiography in certain patients presenting with presumed coronavirus illness. A readily available portable ultrasound machine which could be cleaned after each use was present in the emergency department; the cardiac probe facilitated the acquisition of an echocardiogram at the bedside, without transferring equipment into a coronavirus isolation area. This allowed for an echocardiogram to be obtained 12 hours after the patient had presented to the emergency department, and advocates for the availability of such portable ultrasound machines in all emergency rooms.

\section{REFERENCES}

1. Watkins DA, Johnson CO, Colquhoun SM, Karthikeyan G, Beaton A, Bukhman G, et al. Global, regional, and national burden of rheumatic heart disease, 1990-2015. N Engl J Med 2017;377:713-722. 